\title{
Chemical mechanical modification of nanodiamond in aqueous system
}

\author{
(C) Y.W. Zhu*,**, X.Q. Shen ${ }^{* *, * *}$, B.C. Wang ${ }^{* *}$, X.Y. Xu**, Z.J. Feng* \\ * Department of Precision Instruments \& Mechanology, Tsinghua University, \\ Beijing 100084, China \\ ** China R\&D Center, Changsha Research Institute of Mining and Metallurgy, \\ Hunan 410012, China \\ *** China Department of Materials Science and Technology, Jiangsu University, \\ Jiangsu, 202013 China \\ E-mail: y.w.zhu@63.net
}

\begin{abstract}
The effect of Chemical Mechanical Modification (CMM) on the $\xi$-potential and size distribution of nanodiamonds was studied. Results show significant change in the functional groups on its surface after the CMM treatment by the anionic surface modifier DN-10. The amount of hydroxyl groups decreases and two peaks connected with the stretching vibration of carboxylate appear instead of those of carbonyl. The $\xi$-potential goes up significantly and the size drops to some extent. If treated further with CMM1, a new absorption peak $1382.19 \mathrm{~cm}^{-1}$ connected with the vibration of some distinctive functional groups which cause the increase in the $\xi$-potential and the decrease in size appears.
\end{abstract}

\section{Introduction}

Owing to its excellent mechanical, medical, and electronic characteristics, nanodiamond has been applied in the fields of run-in oil of engine, wear-resistant plating layer of parts, and wear-resistant alloying parts to some extent. It has a great application potential in such sectors, as ultra-fine polishing of silicon wafers and other man-made crystals, modification of plastics and rubber magnetic memory devises and cold cathode display [1-4]. In these applications, dispersion and stability of nanodiamond in various suspensions are the decisive preconditions of its application. When used for ultra-fine polishing of crystals, aggregates may cause nicks on the surface of a work-piece. When used for electroplating or non-electrolytic plating, aggregates result in the inadequate particle distribution and a decrease in the wear-resistant properties.

In aqueous systems, a particle always carries electric charges because of ionization, absorption of ions or preferential substitution of ions [5]. The $\xi$-potential of an ultra-fine particle is often used to evaluate the variety and intensity of charge, and it is a very important index that reflects the repulsive force intensity among particles and the dispersion stability. There exist such functional groups as $-\mathrm{COOH},-\mathrm{OH},-\mathrm{NH}_{2}$, and the like on the surface of nanodiamond [6]. The kinds and intensity of functional groups affect its sorption behavior and ionization properties. The status of charge exerts great influence on the stability of nanodiamond suspensions.

It was pointed out in [4] that the $\xi$-potentials for different fractions of ultradispersed diamond were different.

The Chemical Mechanical Modification (CMM) treatment is a kind of process, which involves particle fragmentation and surface modification at the same time. Good results were achieved by the CMM treatment of calcium carbonate $\mathrm{CaCO}_{3}$ and wollastonite $[7,8]$.
This paper presents some results about the effect of $\mathrm{CMM}$ on the surface functional groups, $\zeta$-potential and size distribution of nanodiamonds.

\section{Experimental}

A grey powder of the nanodiamond sample $L$ (produced by a Chinese corporation Lingyun Nano-materials Co., Ltd) was investigated. Before measuring the $\xi$-potential, an adequate amount of the nanodiamond powder should be dispersed into deionized water to get its suspension. If there is precipitation, the supernatant could be used to measure its $\xi$-potential, as the $\xi$-potential is independent on the particle size. All the $\xi$-potential and most of size distribution measurements were conducted by ZETASIZER3000HS, other size measurements were conducted by small angle $X$-ray scattering (SAXS) and CILAS 1064 Liquid. The primary size distribution was studied by SAXS, which ran at $35 \mathrm{kV}$ and $30 \mathrm{~mA}$. The $X$-ray source was generated by Co $K_{\alpha}$ and the filter was iron. The size parameters such as the mean size, the median size and the distribution can be obtained from the SAXS results. The CMMs of nanodiamond were carried out in a stirring mill. In the process of stirring, the anionic surfactant DN-10 was added into the mill to modify its surface characteristics. The mass ratio of DN-10 to nanodiamond was 5:100 and the peripheral velocity of the stirring vane was about $4.5 \mathrm{~m} / \mathrm{s}$. CMM1 is a further treatment after CMM, which employs the same equipment as $\mathrm{CMM}$ and an more acidic environment. The infrared spectrum analysis in this work was conducted using Nexus 470.

\section{Results and Duscussions}

3.1. $\xi$-potential of sample $L$. The $\xi$-potential with the $\mathrm{pH}$ curve for sample $L$ was presented in Fig. 1 (see curve $a$ ). Its Isoelectric Point (IEP) was found to be at 4.3. 


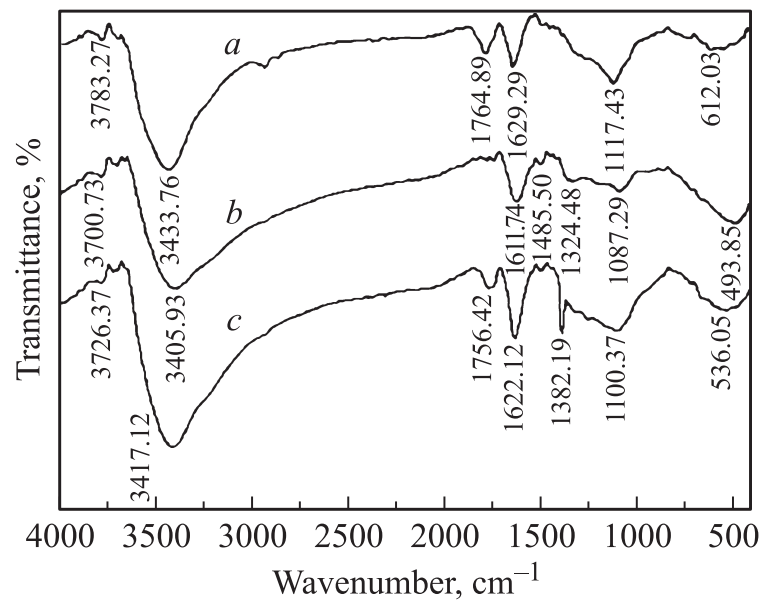

Figure 1. The $\xi$-potential of nanodiamond before and after treatments. $a-$ Sample $L, b$ - after CMM treatment, $c$ - after CMM1 treatment.

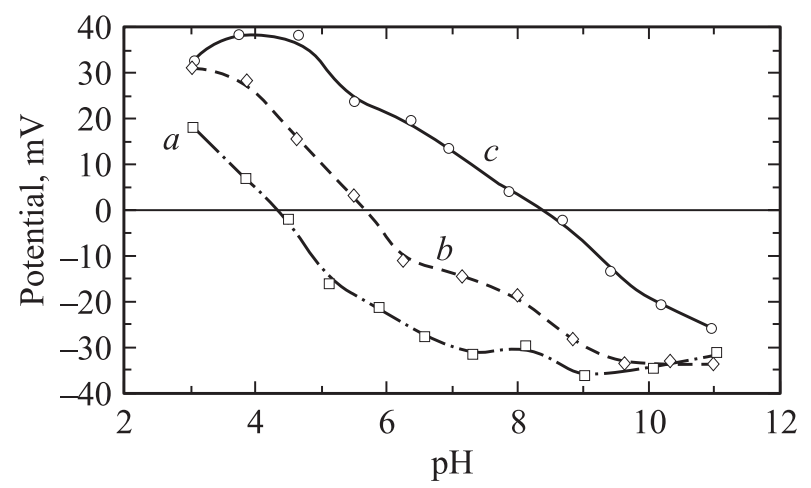

Figure 2. Infrared spectra before and after treatment. $a-$ Sample $L, b-$ after CMM treatment, $c-$ after CMM1 treatment.

The $\xi$-potential is positive in the $\mathrm{pH}$ range of $3-4.3$, and negative in the range of $4.3-11$. Its absolute value is more than $30 \mathrm{mV}$ the $\mathrm{pH}$ range between 7 and 11, which ensures stable suspension without coarse particles.

Infrared spectrum of sample $L$ (see curve $a$ Fig. 2) reveals a very strong absorption peak located at $1117.43 \mathrm{~cm}^{-1}$. It may be connected with the stretching $\mathrm{C}-\mathrm{OH}$ vibration of hydroxyl or/and $\mathrm{C}-\mathrm{N}$ vibration of amine on the nanodiamond surface. It could be reinforced by the absorption of $\left(\mathrm{SO}_{4}\right)_{2}^{-}$or $\left(\mathrm{ClO}_{4}\right)^{-}$during its purification. The absorption peak at $1764.89 \mathrm{~cm}^{-1}$ may be connected with distinctive absorption of carbonyl for carboxyl. There is a very strong absorption peak around $3450 \mathrm{~cm}^{-1}$, it may be induced by the stretching $\mathrm{O}-\mathrm{H}$ or / and $\mathrm{N}-\mathrm{H}$ vibration. Absorption peak locating at $1629.29 \mathrm{~cm}^{-1}$ may be due to the combinational action of I $\vartheta \mathrm{C}=\mathrm{O}$ and II $\delta \mathrm{N}-\mathrm{H}+\vartheta \mathrm{C}-\mathrm{N}$. It also may be induced by the functional group $\mathrm{C}-\mathrm{N}=\mathrm{O}$. It can be suggested from the above analysis and the comparison in absorption intensity among the peaks that there are less $\mathrm{NH}_{2}$ groups and more $-\mathrm{COOH}$ groups on the nanodiamond surface. As a result, the particles showed electronegative in neutral water system. With the rise of $\mathrm{pH}$, the intensity of charge is reinforced because of absorption of $\mathrm{OH}^{-}$and the $\xi$-potential becomes more negative. With the decrease of the $\mathrm{pH}$ value, the $\xi$-potential became more positive until it showed electropositive at the $\mathrm{pH}=4.3$ as a result of the absorption of $\mathrm{H}^{+}$.

3.2. CMMs of Nan odiamond. The $\xi$-potential versus $\mathrm{pH}$ curve after the CMM treatment by $\mathrm{DN}-10$ for sample $L$ was presented by the curve $b$ in Fig. 1 . The curve $c$ in Fig. 1 represented that of chemical mechanical modified sample $L$ by further CMM1 treatment. CMM1 is also a kind of CMM, the purpose of which is to change its surface functional groups.

After sample $L$ treated with CMM and CMM1, the $\xi$-potential with the $\mathrm{pH}$ curve goes up and the IEP shifts to the right. The $\xi$-potential of nanodiamond boosts a lot in the acid range. And it is more than $30 \mathrm{mV}$ in the $\mathrm{pH}$ range of 3-5 for sample treated with CMM1. As a result, the stability of nanodiamond suspension in this range improves greatly. So, the suspension in the above $\mathrm{pH}$ range could be kept for a long time without obvious precipitation.

After the CMM treatment, the absorption peaks at 1629.29 and $1764.89 \mathrm{~cm}^{-1}$ of carbonyl for sample $L$ disappear, substituted by peaks at 1611.74 and $1324.48 \mathrm{~cm}^{-1}$ caused by the stretching vibration of carboxylate. The intensity of absorption peak locating at $1117.43 \mathrm{~cm}^{-1}$ decreases obviously and it shifts to the lower wave number values by $30 \mathrm{~cm}^{-1}$. And this peak is connected with the stretching $\mathrm{C}-\mathrm{OH}$ and $\mathrm{C}-\mathrm{N}$ vibration. The intensity of absorption peak at $3405.94 \mathrm{~cm}^{-1}$ also decreases obviously. The CMM treatment exerted little influence on $\mathrm{C}-\mathrm{N}$ groups, but it could make it easily for some functional groups to be linked to the $\mathrm{C}-\mathrm{OH}$ groups. It suggests that the amount of $\mathrm{C}-\mathrm{OH}$ bonds on the nanodiamond surface drops. It is the drop of the amount of $-\mathrm{OH}$ groups that causes the decrease of intensity of absorption peak. The changes mentioned above are the main reasons why the $\xi$-potential for sample CMM shifts upper in the acid range. The absorption peaks of stretching vibration of carboxylates for sample CMM1 disappears completely, and substituted by peaks of carbonyl again. Compared with sample $L$, a new absorption peak at $1382.19 \mathrm{~cm}^{-1}$ for sample CMM1 appears, in connection with the stretching vibration of some distinctive functional groups, which causes the rise of the $\xi$-potential.

3.3. Effect of CMM on the Size Distribution. After CMM and further treatment (CMM1), the size distribution of sample $L$ changes markedly (listed in Table 1 ). Before the CMM treatment, the fraction of particles less than $100 \mathrm{~nm}$ for sample $L$ is only $0.77 \mathrm{wt} . \%$, and the average size is $2260 \mathrm{~nm}$ and the maximal size is $12000 \mathrm{~nm}$. Here, the particle size refers to the one of the secondary particle, i. e., the aggregate particle size because of various kinds of forces. The results of the SAXS of sample $L$ (listed in Table 2) show that the mean size of primary particles is only $12 \mathrm{~nm}$ and all the particles are less than $60 \mathrm{~nm}$. Here, we refer the particle before aggregation (measured SAXS) to the primary particle. The mean size of the secondary 
Table 1. Size distribution of nanodiamond before and after treatments

\begin{tabular}{l|c|c|c}
\hline \multicolumn{1}{c|}{ Samples } & $\begin{array}{c}\text { Mass fraction } \\
(<100 \mathrm{~nm}), \%\end{array}$ & $\begin{array}{c}\text { Mean size, } \\
\mathrm{nm}\end{array}$ & $\begin{array}{c}\text { Maximum } \\
\text { size, } \mathrm{nm}\end{array}$ \\
\hline Sample $L$ & 0.77 & 2.260 & 12.000 \\
After CMM & 7.57 & 670 & 3.000 \\
After CMM1 & 81.6 & 95.6 & 205.6
\end{tabular}

Table 2. Results of SAXS for sample $L$

\begin{tabular}{c|c|c}
\hline Size interval, $\mathrm{nm}$ & Mass fraction, $\%$ & Cumulative, $\%$ \\
\hline $1-5$ & 20.1 & 20.1 \\
$5-10$ & 43.0 & 63.1 \\
$10-18$ & 25.2 & 88.3 \\
$18-36$ & 4.7 & 93.0 \\
$36-60$ & 7.0 & 100
\end{tabular}

Table 3. Size distributions after the addition of gallic acid

\begin{tabular}{c|c|c}
\hline Size range, $\mathrm{nm}$ & Volume, $\%$ & Number, $\%$ \\
\hline $17.8-21.8$ & 0 & 0 \\
$21.8-26.6$ & 3.2 & 10.5 \\
$26.6-32.5$ & 10.6 & 22.8 \\
$32.5-39.7$ & 19.8 & 28.4 \\
$39.7-48.5$ & 24.3 & 21.0 \\
$48.5-59.3$ & 23.0 & 11.8 \\
$59.3-72.5$ & 16.6 & 5.4 \\
$72.5-88.5$ & 2.5 & 0.1 \\
$88.5-108.5$ & 0 & 0
\end{tabular}

particle measured by ZETASIZER3000HS is 100-200 times coarser than that of SAXS, namely, the index of aggregation of nanodiamond sample $L$ is $100-200$.

The formation and properties of nanodiamond aggregates are very complicated. Some are soft aggregates caused by van der Waals forces among particles, some are hard ones caused by chemical bonds among particles. It is very difficult to disperse them, especially for the latter. Detonation is a very complicated transit high pressurehigh temperature process involved many chemical reactions. Under those conditions, carbon droplets are easily conglutinated in the cooling and phase transformation process, then hard aggregates form. Nanodiamond has a large specific surface, which results in a huge surface energy. In order to lower the free energy of the system, the fine particles congregate to aggregates with a large size spontaneously. The obvious improvement of the nanodiamond size distribution is observed after the CMM treatment: ithe mean size of sample drops from 2260 to $670 \mathrm{~nm}$. And after further treatment (CMM1), it drops further to $95.6 \mathrm{~nm}$, and most particles are finer than $100 \mathrm{~nm}$.
Under usual stirring grinding conditions, smaller particles are formed due to the media grinding and shearing action. But those smaller particles easily congregate to be larger ones as a result of the demand of reducing the free energy of the system once the stirring stops. So, usual stirring grinding has little effect on the decrease of the size for ultrafine particles. The reason why the nanodiamond aggregates can be effectively crushed during CMM process may be attributed to the combinational action of mechanical grinding and chemical absorption during the stirring grinding. With the addition of DN-10 in the process of stirring grinding, smaller aggregates form due to the grinding of media and shearing action, and at the same time the temperature of the nanodiamond aggregate surface rises and its activity improves, which results in the reaction between DN-10 molecules and the active spots on nanodiamond aggregates. The surface electrical properties change markedly because of the chemical absorption of DN-10. The $\xi$-potential of nanodiamond particles increases a lot in the $\mathrm{pH}$ range between 3 and 7, and the enforced repulsive forces among particles protect them from aggregating. As a result, the mean size of nanodiamonds drops.

After sample $L$ treated with CMM and further treatment CMM1, its particle size could be further reduced with the addition of $0.5 \mathrm{mg}$ gallic acid per liter. Table 3 presents its size distribution. No particles with the size more than $88.5 \mathrm{~nm}$ are found. The mean volume size of nanodiamond is $52 \mathrm{~nm}$. And the suspension can be used for ultra-fine polishing of crystals and composite electro-less plating and electroplating of parts.

\section{Conclusions}

1) After treated with $\mathrm{CMM}$, the amount of $-\mathrm{OH}$ groups decreases, if further treated with CMM1, a new absorption peak at $1382.19 \mathrm{~cm}^{-1}$ appears which is connected with some distinctive groups. The $\xi$-potential goes up in the acid range, and the stability of its suspension improves a lot.

2) After treated with CMM and CMM1, the average volume size drops from 2260 to $95.6 \mathrm{~nm}$. If further dispersed with the addition of $0.5 \mathrm{mg}$ gallic acid per liter, the mean volume size reaches $52 \mathrm{~nm}$.

\section{References}

[1] G.Z. Wang. Proceeding of the Symposium of Development of Nanodiamond. Hangzhou (1999). P. 7.

[2] W. Zhu, G.P. Kochanski, S. Jin. Science 282, 1471 (1998).

[3] N. Kossovsky, A. Gelman, H.J. Huatyszyn et al. Bioconiugate Chemistry 6, 507 (1995).

[4] V.Yu. Dolmatov. Russian Chem. Rev. 70, 7, 607 (2001).

[5] F.S. Li. Technologies for Ultra-fine Particles. Changsha: National Industrial Publishing, House (2000). P. 7.

[6] E. Mironov, A. Koretz, E. Petrov. Diamond and Related Materials 11, 872 (2002).

[7] H. Ding, Y.S. Han. Non-Metallic Mines 4, 32 (1997).

[8] L. Li, X.B. Zheng. Non-Metallic Mines 5, 13 (1993). 